\title{
The Influence of Lumbar Stabilizing Orthoses (Lumbamed Plus Pushing Application; Fa. Medi Bayreuth) in Lower Back Pain. A Prospective Randomized Study about Pain Relief, Muscular Strength and Posture of 70 Patients
}

\author{
Rene Thomas* \\ Department of Orthopedics, Germany \\ *Corresponding author: Rene Thomas, Department of Orthopedics, Germany
}

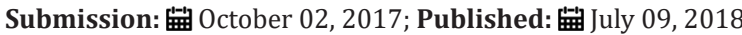

\begin{abstract}
Lumbar pain is very common problem in elderly patients in the western world and mostly the reason for inability to work. Influence of orthopedic devices on these patients is very controversial in orthopedics and physical therapy as effect on the posture was not mentioned yet and even a negative effect on the muscle strength of abdominal and lumbar muscle was respected. The aim of this study was to assess the effect of lumbar orthoses on the pain, muscular dysbalance and posture as well as on strength in comparison to natural history without specific treatment and with physical therapy. The prospective randomized study was done with 70 patients with pain over a period of 6 weeks.
\end{abstract}

4 groups were tested:
A. No therapy,
B. Only lumbar orthoses,
C. Only physical therapy,
D. Physical therapy plus orthoses.

The examination consisted in questionnaire about back pain (Bessel analog scale) amount of medicaments taken, general state of health, isometric maximal muscle testing and posture survey with computer surface measurements (Optrimetrie). In the first two weeks the groups with lumbar orthosis showed significant as pain reduction and reduced pain killing drugs and kept this for the post study period of 6 weeks. Muscle strength progressed in the groups proved by orthoses. The posture did not change very much in the study period whereas the reduction of lumbar lordosis was the highest in the group with lumbar orthoses and physical therapy.

Conclusion:Stabilisation orthoses enable early reduction of pain and mobility as well as the improvement of muscle strength. The old theory about muscle weakness due to lumbar orthoses has to be abolished. Lumbar orthoses are low-cost and effective treatment for lumbar back pain and can definitively added to physical therapy to improve in short time period muscle strength and posture and brings patients back to live.

Keywords: Low backpain; Spinal disorders; Muscle strength; Lumbar belt; Physiotherapy

\section{Introduction}

From a functional point of view, it can be assumed that in about $80-90 \%$ of cases back pain originates in the (arthro) muscular system. Stress acts as a co-factor. Patients suffering from back pain, for example, often have significantly reduced trunk muscle strength and altered muscle conditions compared to people who do not suffer from back pain [1-6]. The problems of recognizing craniomandibular dysfunction (CMD) or functional pelvic obliquity and the resulting back pain are well known. However, the objective diagnosis of such diseases is often a challenge.

The typical clinical picture of lumbago often impresses as a so-called sciatica scoliosis, which corresponds to a myogelosisinduced bodily abnormal statics. Depending on age, radiological changes can almost always be found, which should, however, primarily be understood as physiological adaptation of the axial 
organ to misalignment and overload. There is often a risk of treating X-ray or MRI findings. The therapeutic approach aims to quickly eliminate painful muscle tension by means of conservative measures (medicines, physiotherapy, "bandages", medical training therapy, sports). The prerequisite for participation in targeted therapy programmes is first of all rapid pain relief. Only in this way can the risk of chronification be effectively reduced. Unfortunately, there is often a lack of uniform therapy regimens in the treatment of back pain, so that in many cases a prolonged "pain osteopathy" is the result.

Apart from a quick relief of pain by injections, NSAIDs and physical measures, the longer-term solution of the "back pain problem" can only be achieved by an improvement of the false statics with consecutive incorrect strain on the spine. The spinal stabilizing muscles play a decisive role in this process. Even from our own arduous experience, everyone knows that only targeted and intensive strengthening exercises can bring success here. Patients with back pain can be even more difficult to achieve what healthy people sometimes find difficult. Stabilizing orthoses (formerly known as lumbar bandages) are a proven means of quickly and effectively correcting spinal disorders.

The question of whether this has a positive effect on the spine via the abdominal press and the associated increase in intraabdominal pressure, or whether the additional heat effect on the muscles contributes to pain relief, cannot be answered with absolute certainty. All in all, clinical practice shows predominantly positive effects with regard to pain reduction. The effect of stabilization orthoses on the torso-stabilizing musculature was not quite clear until now. For decades now, the opinion has been that stabilization orthoses should only be worn temporarily, as the musculature becomes even more insufficient under therapy. Unfortunately, objective measurement data regarding the change in muscle strength are missing.

An essential subject of the investigation was the question of whether stabilizing orthoses with regular use have a demonstrable effect on body failure. In particular, it was to be determined in which form the performance of the body-stabilizing musculature changes. For this purpose, the correlation of subjective parameters, such as pain and general well-being with clinically measurable force and static values in the various patient groups. In addition, the question of the influence of a twice-weekly device-supported spinal gymnastics (12x total) was investigated.

\section{Material and Methodology}

The present prospective study included 98 patients aged 37 to 69 years of age who were presented in the orthopaedic practice due to an acute exacerbation of chronic back pain (pain episodes at irregular intervals of at least 3 months) in the orthopaedic practice. This was exclusively pseudo-radicular lumbar pain (Lumbago) without sensomotorical deficits. The observation interval was set at 6 weeks. The 98 patients were initially divided into an orthosis group and a non-orthosis group by block randomization. In the two resulting groups (with and without bandage) half of the patients received 12 times device-supported physiotherapy. The additional physiotherapy should determine whether the strength values in the active groups are statistically significantly better than in the groups without physiotherapy. Of particular interest was the question of whether the lumbar bandage was worn at an early stage and whether the complaints were more regressive. Of the 98 patients who were primarily included in the study, 70 patients completed the post-marketing observation completely. All patients received a pain diary (Mundipharma) in which the degree of pain (VAS 1-10) should be entered over a period of 6 weeks $4 \mathrm{x}$ daily. At the first presentation due to back pain, Ibuprofen $6003 \times 1$ daily was prescribed as a standard therapy regimen for 3 days in all patients, as long as no gastrointestinal contraindications existed. If there was no satisfactory pain relief afterwards, Tramal drops were added for a maximum of one week (Figure $1 \& 2$ ).

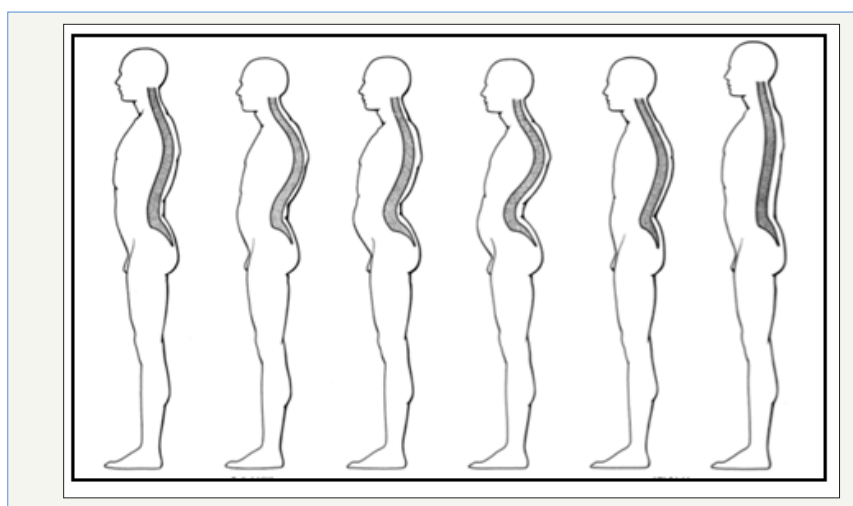

Figure 1: Posture types and their significance for the development of back pain. Both hyperlordoses and especially patients with a raised lumbar lordosis (steep position) are predestined for back pain (23).

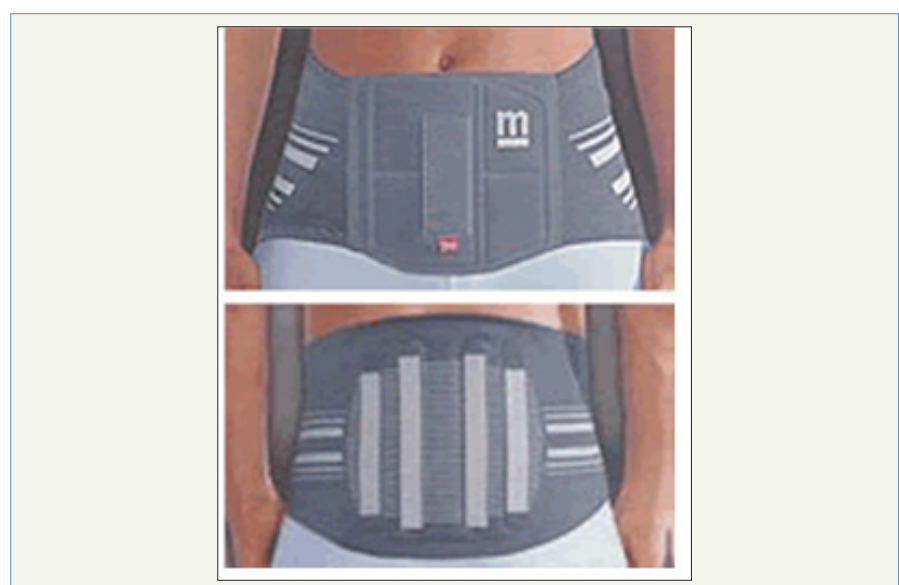

Figure 2: Lumbamed lumbar spine stabilization orthosis.

After alleviating the acute pain, a 3D vertebral column analysis and isometric force testing were carried out after about one week in all patients. The patients of groups 3 and 4 started wearing the Lumbar Stabilization Orthosis immediately after the first presentation. As an average wearing time of 6-8 hours per day, especially when standing and walking were agreed upon. In order to optically verify the influence of the lumbar spine stabilization orthosis on the body's malformed statics, a Upright-MRI investigation of a 56 year old volunteer with known pseudoradicular 
lumbar pain was carried out experimentally. Morphologically, a significant correction of the lumbar hyperlordosis was observed with the stabilization orthosis in place. The lordosis angle without orthosis was? and with orthosis? Degrees (Figure 3).

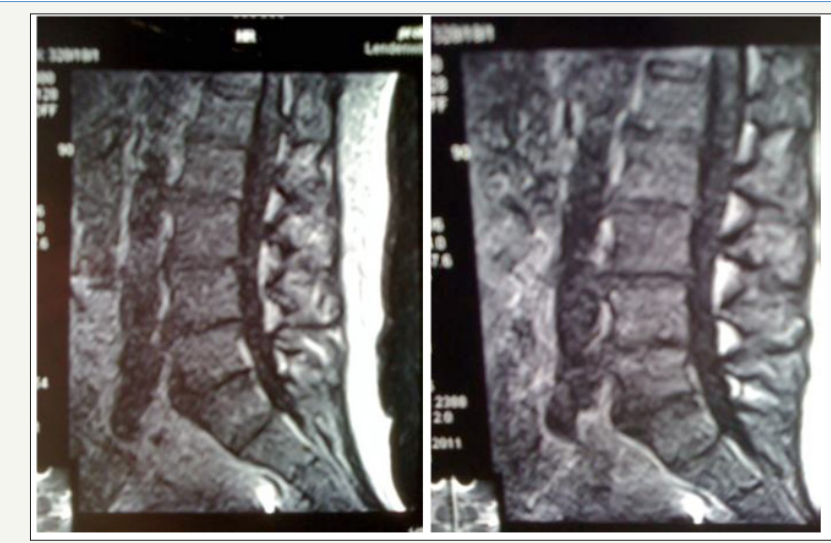

Figure 3: Example 56-year-old patient left and right with lumbar spine stabilization orthosis with a lower degree of hyperlordosis.

\section{D Spine Analysis}

After one week and under the condition of sufficient painregeneration for further diagnosis, all patients underwent a 3-D spinal measurement (Company DIERS). Prior to this, it was clinically verified that no pain-related malpositions, such as sciatica, were present. By means of a trigonometric method (optrimetry) the spine is measured by means of a surface grid and various functional parameters are ascertained. In general, the unclothed patient was measured standing without stabilization orthosis. The measurement was then repeated after the observation interval of 6 weeks.

\section{Isometric Measurement of Muscle Strength (Tergumed)}

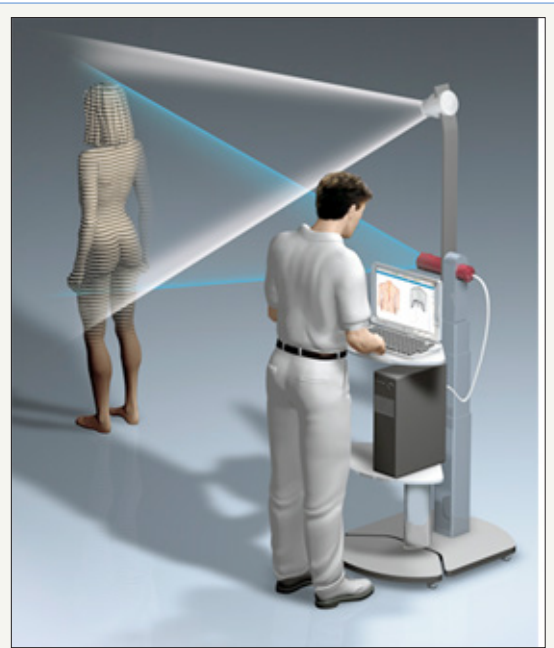

Figure 4: A line grid projected onto the back of the body is recorded by means of a video camera and the spinal measurement is then carried out by means of triangulation.

Isometric force measuring and training devices from PROXOMED (TERGUMED) were used to verify the muscular strength and to detect imbalances in the area of the torso-stabilizing musculature. Within the scope of the standardized testing, the isometric muscle strength of the abdominal musculature (flexion), back stretcher (extension) and the lateral tilt (right/left) were measured summatorically (Figure 4-8).

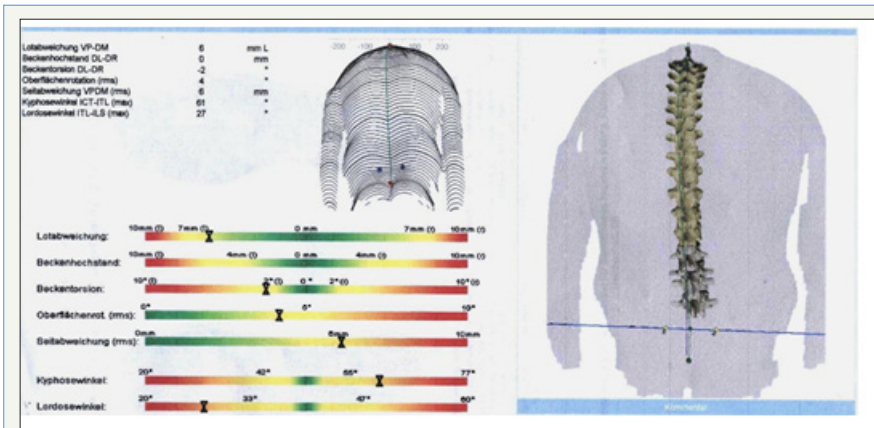

Figure 5: Example of a measurement printout (excerpt with total overview of dorsal) In addition to the sagittal profile, which is of primary interest, the spinal column statics were also evaluated in the frontal plane.

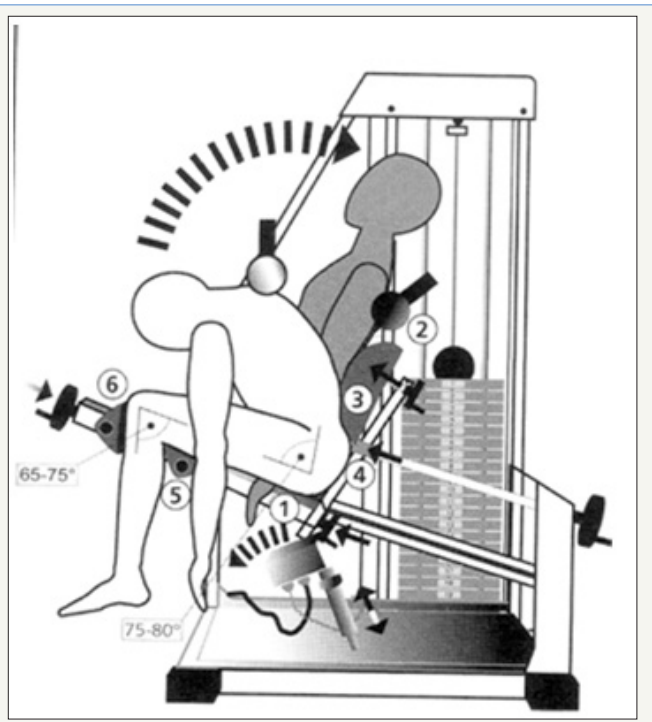

Figure 6: measured values after 3-D spinal analysis.

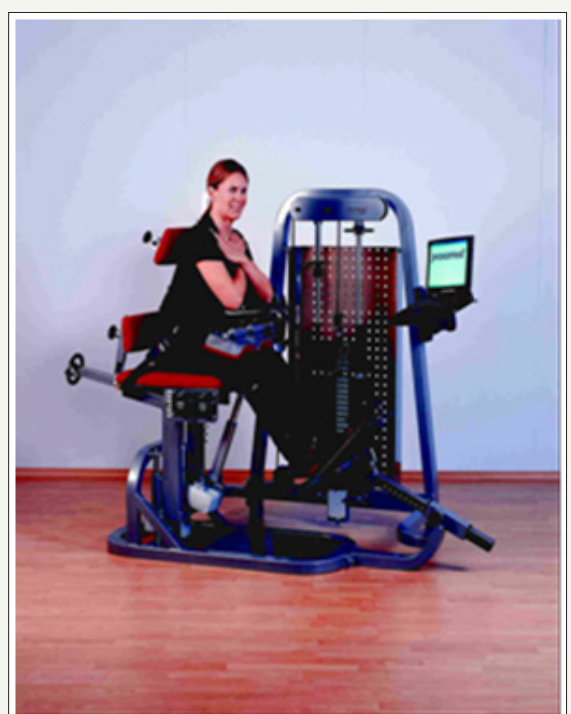

Figure 7: Tergumed measuring system (example extension). 


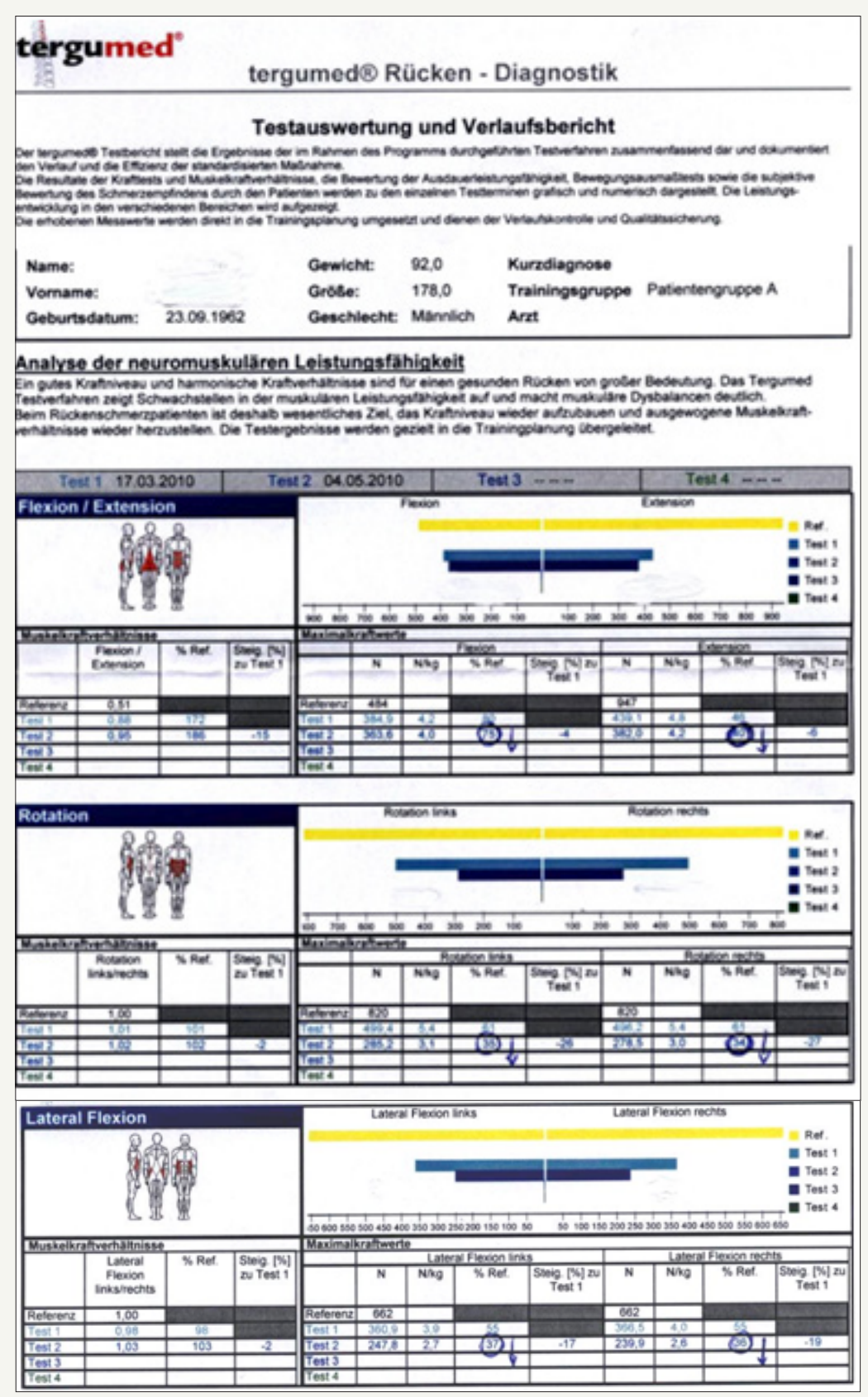

Figure 8: Results overview after isometric muscle testing. Pictured patient values (group 1A) with Deterioration of the force values after 6 weeks (dark blue bar). In addition, patients were evaluated by means of a questionnaire from the Research and Prevention Center Cologne (FPZ) regarding their subjective condition before and after the 6 weeks. Muscle testing was also performed only after adequate pain regression.Prevention Center Cologne (FPZ) regarding their subjective condition before and after the 6 weeks. Muscle testing was also performed only after adequate pain regression.

\section{Results}

When considering the results, it should be borne in mind that the examined patients were a very heterogeneous group of men and women of different age groups and that the problem of "back pain" is caused by multiple factors. The collected parameters can be used as a trend statement because of the relatively small patient groups, further more extensive examinations should follow at intervals in order to further substantiate the results. Pain progression (pain diary) The statistical evaluation of the pain curves (VAS) was carried out by summing up the individual values [1-11] collected four times daily in the morning, at noon, in the evening, at night in the sense of a daily total value. This could result in maximum pain 40 (4x10). This is an artificially formed parameter for evaluation purposes. The figure shows the comparison of the individual pain curves in the groups with and without stabilization orthosis. This showed a statistically significant decrease of back pain in the orthosis groups at the observed interval. In the non-orthotic group pain values remained at a significantly higher level and fell only slightly. By the end of Week 1, the pain levels in both groups were similar [12].

Subsequently, the intensities stagnated in the non-orthotic groups, while they still fell significantly with orthosis. It can be seen 
from Figure 9a that the daily averages of pain values for groups of subjects with stabilization orthosis (groups 3 and 4) decrease significantly faster compared to groups 1 and 2 without orthosis. The very good data from the pain diaries allow us to carry out detailed evaluations for all groups 1 to 4 and to check for each individual group (intra) whether the reduction in the daily mean of the pain values is significant and to further check by means of a statistical group comparison (inter) which of the therapies is most effective for groups 1 to 4 . The daily averages of the pain values for each of the groups 1 to 4 for the first 10 therapy days are included in the calculations as input values and for the last 10 therapy days as output values [13]. The following Figure $9 \mathrm{~b}$ provides an overview of the daily averages of pain in all groups for the entire therapy period of 42 days. Here a line graphic was selected, because a bar graphic becomes too confusing. Negative output-minus input values in the diagrams indicate an improvement of the condition.

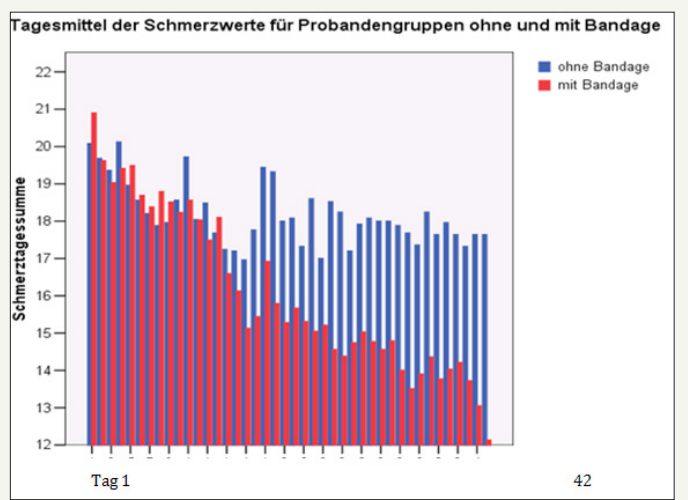

Figure 9a: Daily averages (values of the VAS values were ascertained in $4 \mathrm{x}$ daily the VAS values and a sum of the Individual values formed; max. possible summatory pain value 40).

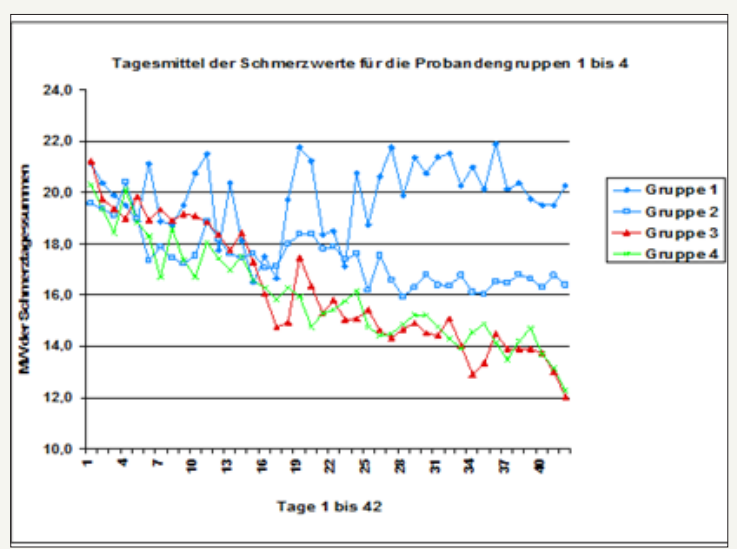

Figure 9b: Differentiated examination of pain levels in the individual groups drug consumption In the groups with stabilization orthosis, there was a statistically significantly lower drug consumption than in the comparison groups without orthosis. In this case, the amount of medication consumed fell particularly sharply until the end of the third week.

\section{Statistical significance of the improvement of sensitivi-} ties

In summary, Group 3 (stabilisation orthosis without a KG) showed the most statistically significant improvements in the regularity and intensity of pain and well-being. This also applied to the intensity of pain in group 4 (stabilisation orthosis and $\mathrm{KG}$ ) (Figure 10-14). In Group 2 (only KG) performance and well-being also improved with a high significance. Performance clearly also improved in Group 4, i. e. this effect was only evident in the KG groups. This can also be assumed for Group 3, but this fact can only be used statistically as a trend statement [14].

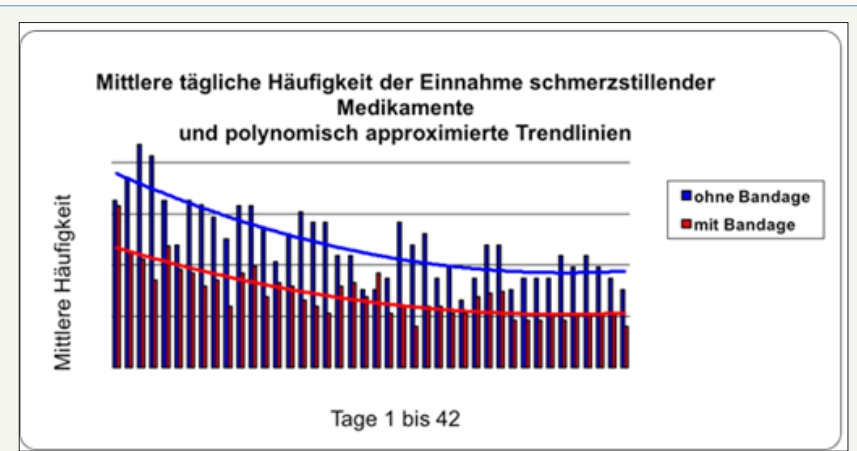

Figure 10: Comparison of the use of analgesics in the groups with and without stabilization orthosis Improvement of the sensitivities The results of the FPZ questionnaire (complaints: regularity of 1 symptom-free to 4 permanent and intensity of 1 symptom-free to 10 unbearable, performance (ability) from 1 very good to 5 very bad and well-being from 1 very good to 4 bad).

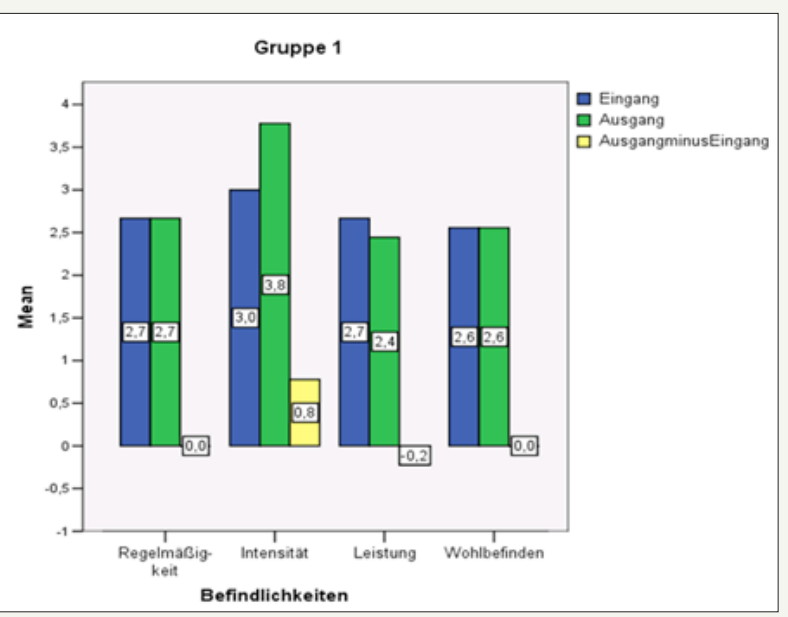

Figure 11: Overall overview of the results in group 1.

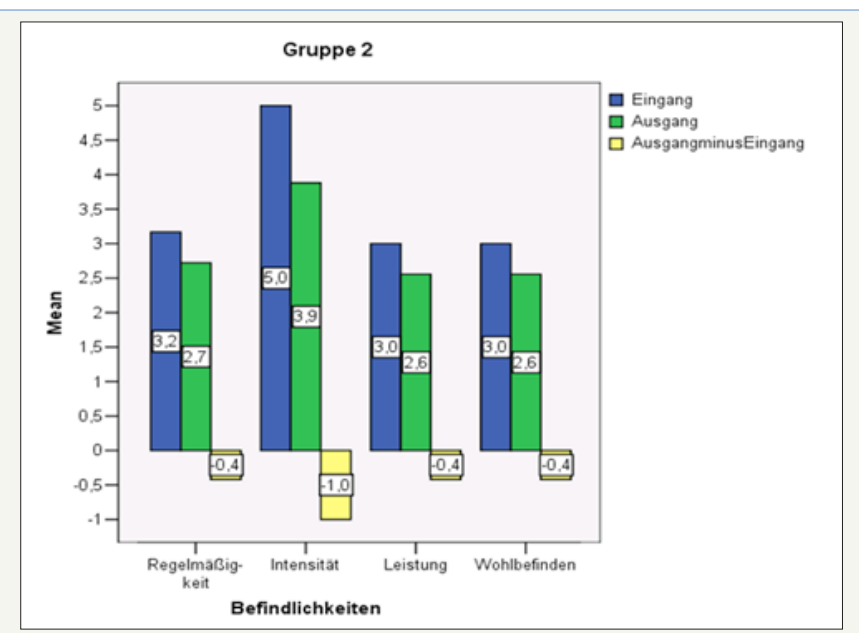

Figure 12: Overall overview of the results in group 2 . 


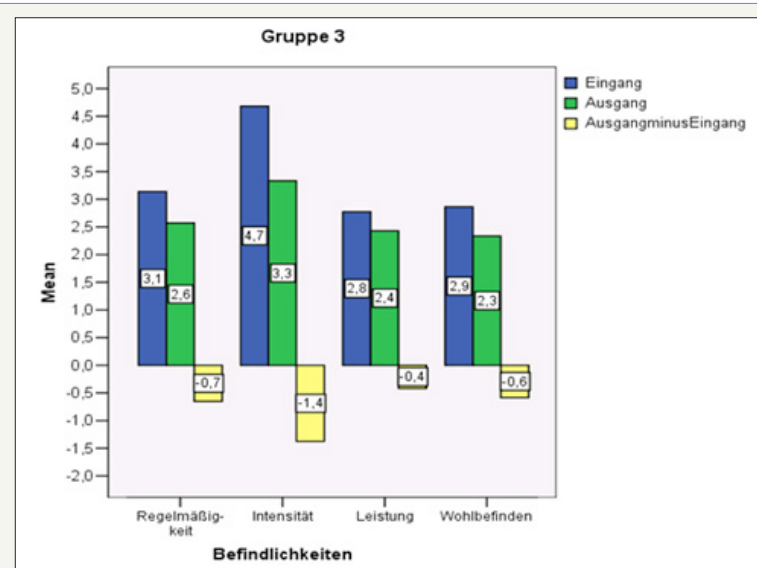

Figure 13: Overall overview of the results in group 3.

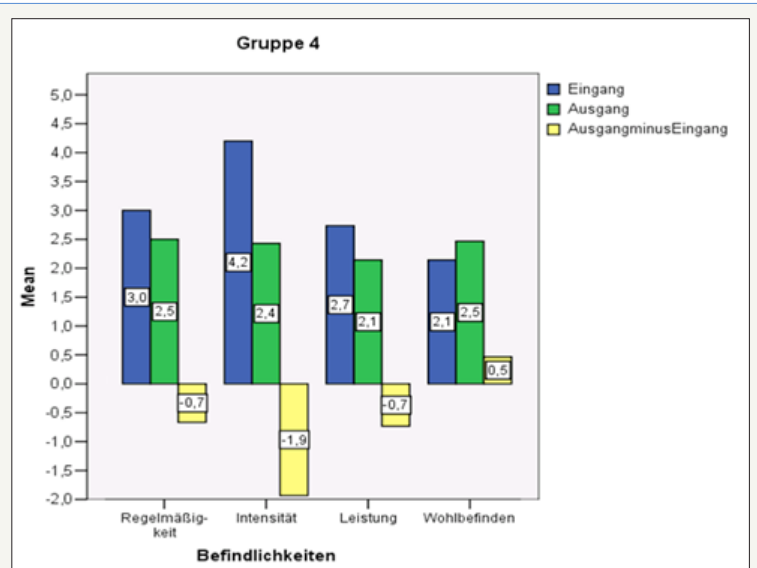

Figure 14: Overall overview of the results in group 4.

\section{Isometric muscle strength testing}

Tables 1: Classification of groups.

\begin{tabular}{|c|l}
\hline Group 1 without orthosis no physio $K G(n=12)$ & Group 3 with orthosis no physio $(n=25)$ \\
\hline Group 2 without orthosis $12 x$ physio $(n=19)$ & Grou 4 with orthosis 12x physio $(n=14)$
\end{tabular}

Tables 2: With the significance levels, which are also in the following studies: Significant: Error probability $<=0.05$ Very significant: Error probability $<=0.01$ Tendency: Probability of error $<=0.1$.

\begin{tabular}{|c|c|c|c|c|}
\hline & Group1 & Group 2 & Group 3 & Group 4 \\
\hline Regelmäßigkeit v. Beschwerden & Keine Signifikanz & Tendenziell Signifikant & sehr signifikant & Tendenziell Signifikant \\
\hline Intensität v. Beschwerden & Keine Signifikanz & Tendenziell Signifikant & sehr signifikant & sehr signifikant \\
\hline Leistung & Keine Signifikanz & Signifikant & tendenziell signifikant & Signifikant \\
\hline Wohlbefinden & Keine Signifikanz & Signifikant & sehr signifikant & Keine Signifikanz \\
\hline
\end{tabular}

At the beginning and after 6 weeks the muscular strength was investigated by using isometric test and therapy devices from Proxomed (TERGUMED) at the beginning and after 6 weeks (Table 1 $\& 2)$. The data collected were additionally validated with reference values from the Research and Prevention Centre Cologne (FPZ). In addition to the extension and flexion, the right and left side tilt hands were also tested. The following results were obtained in the individual groups [15].

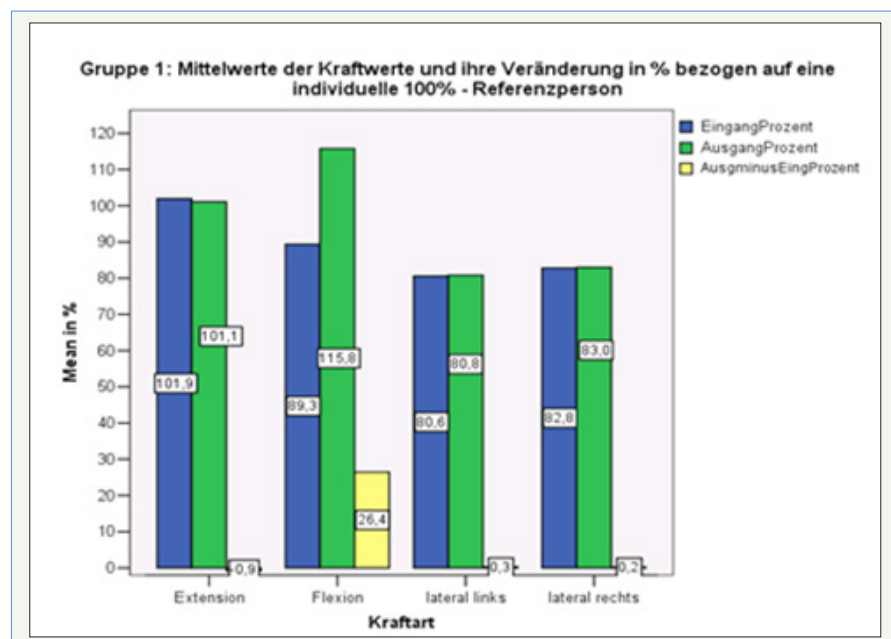

Figure 15: Overall overview of the measured force values in group 1 at the beginning and end of the test. after 6 weeks.
Group 1: (without stabilization orthosis/ without KG) In all directions of motion, the force values did not differ significantly from input and output measurements. Only with flexion was an increase in strength after 6 weeks measurable (Figure 15).

Group 2: (without stabilization orthosis / with 12xKG) increased strength in all directions of motion after 6 weeks, only statistically significant in the case of extension and lateral tilt (Figure 16).

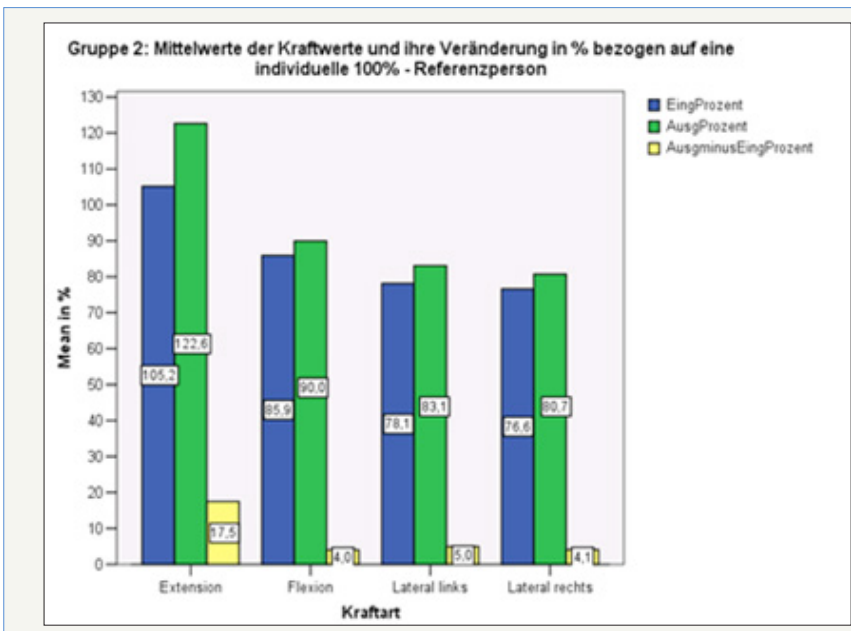

Figure 16: Overall overview of the measured force values in group 2 at the beginning and after 6 weeks. 
Group 3: (with stabilizing orthosis / without KG) In all directions of movement, a statistically significant increase in strength despite bandage was also detectable without KG (Figure 17).

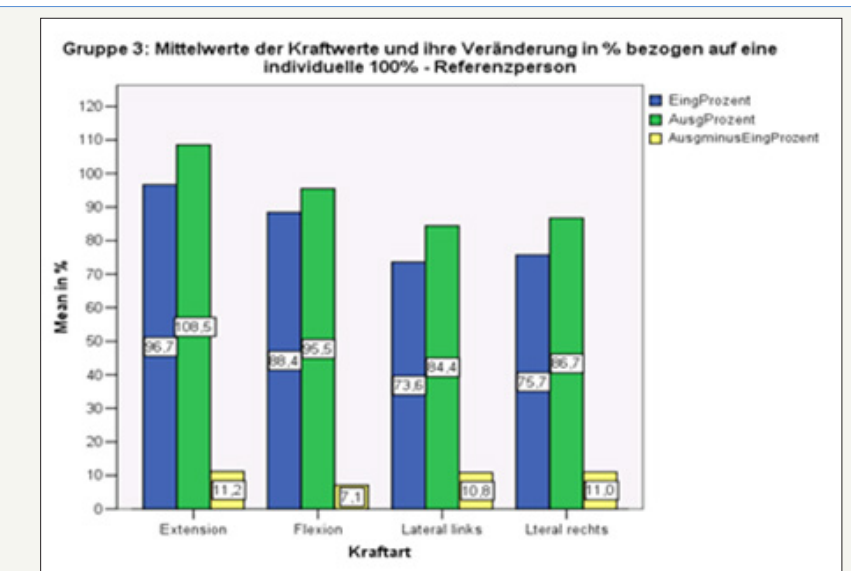

Figure 17: Overall overview of the measured force values in group 3 at the beginning and end of the test. after 6 weeks.

Group 4: (with stabilization orthosis / with 12xKG) In extension and flexion statistically significant increase in force with already very high force values during input measurement. Overall highest values of all groups. The lateral tilt also showed an increase in strength after 12 times physiotherapy. The increase in force was stronger than in Group 2 (Figure 18).

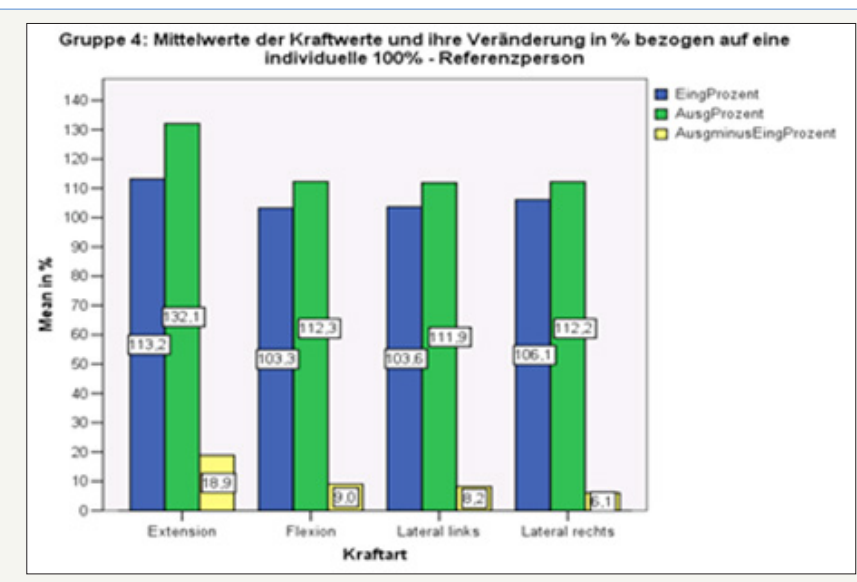

Figure 18: Overall overview of the measured force values in group 4 at the beginning and end of the test. after 6 weeks.

Below, the degrees of force in the individual directions of movement are examined in more detail. For reasons of clarity and under the aspect of the decisive function in securing the sagittal profile, the emphasis is placed on back flexors and extensors and the results are displayed graphically [16-18].

Extension: With the exception of Group 1, in each individual group 2,3 and 4 there are 2,3 and 4 according to the number of participants completed. Training units show a statistically significant percentage increase in strength. In Figure 19, Group 3 shows a significant improvement in the strength of the spine extensors. The changes in strength gains in groups 2 and 4 were not statistically significant. Compared to Group 3 , however, the increase in strength was significantly higher (11.2 to 18.9). It was important to realize that muscle strength also increased in the orthosis groups. In the "physiotherapy groups" the increase in strength was generally the greatest [19].

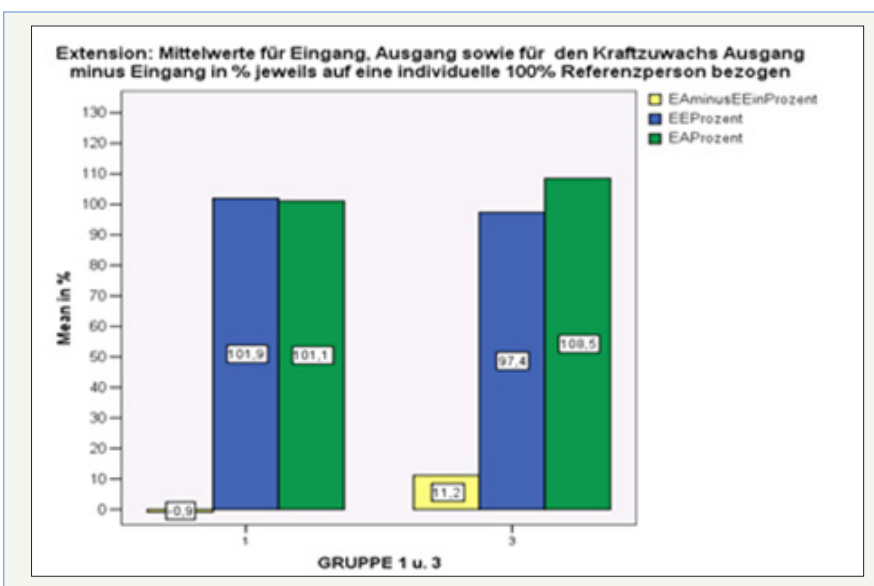

Figure 19: Comparison of results for extension in groups 1 and 3 without KG.

Flexion: Especially when it comes to the problem of securing the sagittal profile, the abdominal muscles are of crucial importance. This is often insufficient or underdeveloped. The measured baseline values in \% were significantly lower compared to the back extensors. The following figures show the results in detail. Group 4 came out on top. SEITNEIGUNG: For reasons of clarity, there is no graphical representation of the results at this point. For the RIGHT inclination, the increase in strength in group 2 was significant, and very significant in group 3 . In groups 1 and 4 there was no significant increase in strength. No statistically significant increase in force was found in any of the groups with a LEFT inclination. Group 3 came first, followed by Group 4 and then Group 2.

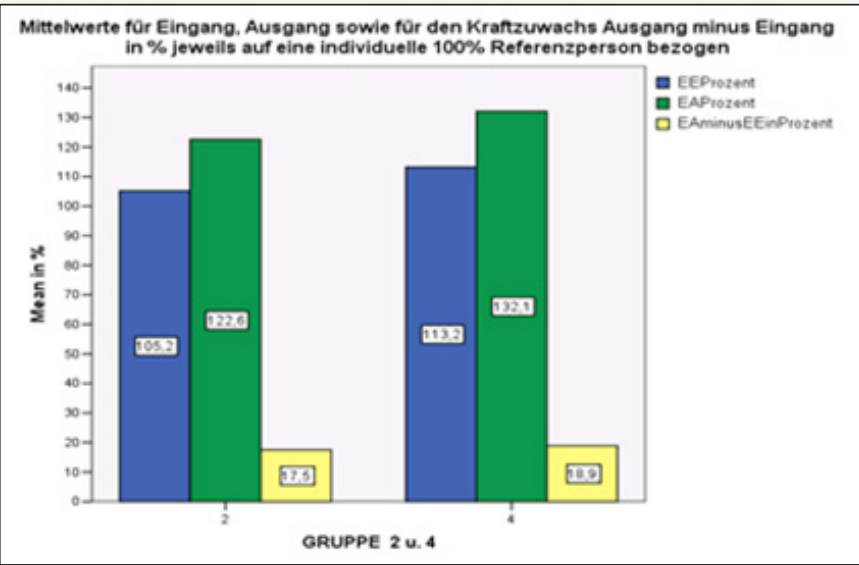

Figure 20: Comparison of results for extension in groups 2 and 4 with KG. Compared to the "non-KG groups", the strength of the spine extensors improved significantly, with a stronger increase in group 4.

In summary, the stabilization orthosis groups ( 3 and 4 ) showed the greatest increase in strength. Except for the back extension, the stabilization orthosis groups generally performed better in terms of the measured increase in strength (Figure 20). 3-D spinal column analysis: The performed optical static analysis provided 7 individual parameters for each patient, which was considered in their entirety. The measuring parameters KW and LW were of particular interest, as these spinal column disorders help to characterize the spinal 
column and thus help to describe the sagittal profile. Explanation of terms: $\mathrm{LA}=$ Solder deviation $\mathrm{Bra}=$ basin height $\mathrm{BT}=\mathrm{Basin}$ torsion $\mathrm{OR}=$ spinal rotation $\mathrm{SA}=$ lateral deviation $\mathrm{KW}=\mathrm{kyphosis}$ angle LW=angle of lordosis First, the overall parameters are displayed in groups and individual parameters are examined in more detail. Details of the statistics in Annex 3. In group 1, the sagittal profile (LW/KW) did not change after 6 weeks. Only the balance (LA) has been improved. Under KG the sagittal profile (LW/KW) improved slightly, the balance worsened. The kyphosis angle corrected less than the lordosis angle. (-0.5 at -0.7). As in Group 2, a slight improvement of the sagittal profile. While the LW hardly benefited $(-0.2)$, the strongest changes in the KW (-0.6) were seen [20-22].

Angle of kyphosis: Only in Group 4 did the angle of kyphosis tend to change for the better. In all other groups except group 1 only slight improvement of the value. Descriptively, these are shown below without a graphic (Figure 21).

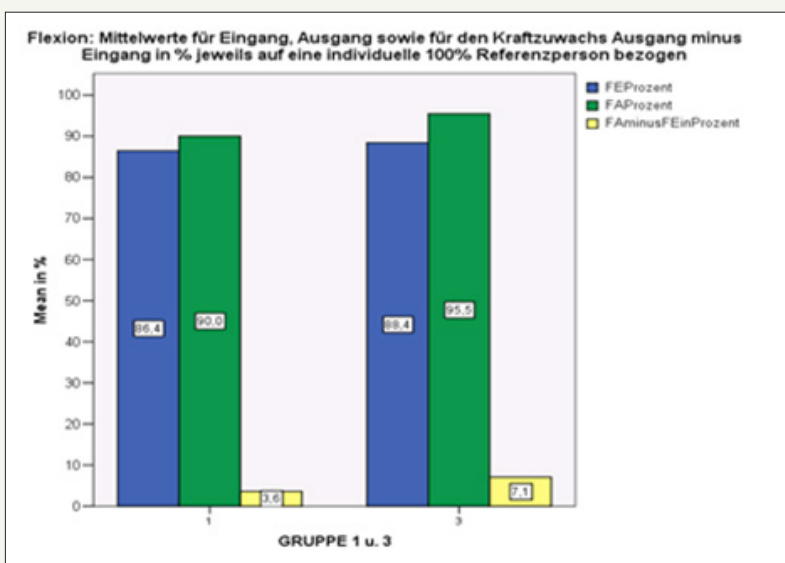

Figure 21: Comparison of the results for flexion in groups 1 and 3 with KG. In stabilization orthosis group 3, the force measured after 6 weeks increased by about twice that of group 1 (3.6 to 7.1 ).

Solder deviation: In groups 1 and 3, there was a reduction of the plumb line deviation after 6 weeks, whereas in the KG-groups it increased. It did not matter whether a stabilization orthosis was worn or not. No significance demonstrated. Pelvic obliquity: The pelvic obliquity decreased only in groups 1 and 2 without stabilization orthosis. In groups 3 and 4 he remained about the same. No significance.

Pelvic torsion: The strongest changes in pelvic torsion were found in group 2. Group 1 also improved, with Group 3 getting worse and Group 4 showing no change after 6 weeks. No significance. Vertebral surface rotation: No statistically relevant changes detected. Group 2 tends to show the greatest improvement, followed by Group 3, with a worsening in 1 and 4 .

Summary statics: While the overall statics did not change significantly within the 6 weeks under consideration, the lumbar angle (described as pelvic tilt) important for lumbar pain has been improved (delordosis). The change was most pronounced in Group 4 with stabilization orthosis and KG, followed by Group 2 (KG) with lower changes.

\section{Discussion}

The recommendation to my patients to wear the stabilization orthosis for longer periods of time every day while walking and standing often drew criticism from physiotherapists and other colleagues. My recommendation resulted mainly from the personal impression that many backache sufferers said it was subjectively better subjectively, if they regularly wore the stabilization orthosis in the everyday activities. The result was that the patients were very unsettled by different opinions. The main argument of the "stabilizing orthotic opponents" was the declining muscle strength by relieving the same. Historically speaking, this reasoning dates back to an era in which the bodice products used were often used rigidly (bar lattice bandages) and superficially immobilizing. Today's modern stabilizing orthoses, on the other hand, have a stabilizing, supportive and balancing effect on the musculature. This is an important starting point in the treatment of back pain (Figure 22). The abdominal press and the resulting improvement in statics have a positive influence on the three most important causes of back pain; reduced muscle strength, muscular imbalances and deficient neuromuscular control. Experience has shown that many patients prefer "light stabilization orthoses" without extensive tension straps and stiffeners in daily practice. The locking mechanism has an important function. Especially when sitting, too rigid velcro systems cause pressure problems in the groin and lower abdomen area. The stabilizing orthosis type (Lumbamed) used in the context of the post-marketing observation already has an improved ergonomic design with a new closure system, which was assessed as very positive by the patients during the final examination (Figure 23).

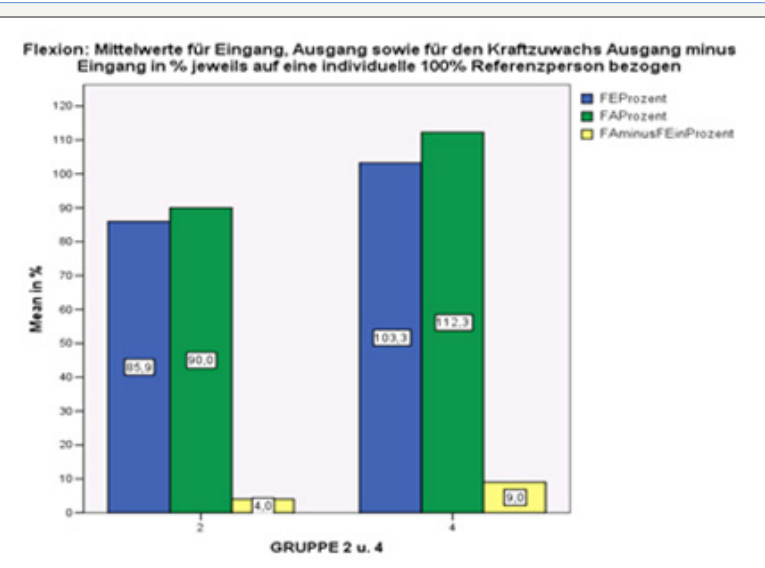

Figure 22: Comparison of results in Flexion in groups 2 and 4 with KG. Generally, only groups 3 and 4 with stabilization orthosis showed a statistically significant increase in strength.

\begin{tabular}{|ll|}
\hline Extension & $4>2>3>1$ \\
Flexion & $4>3>2>1$ \\
Solder deviation right & $3>4>2>1$ \\
Solder deviation left & $3>4>2>1$ \\
\hline
\end{tabular}

Figure 23: Overview of the quantitative increase in force in groups 1-4 in the individual movement directions. 
In the literature, the effectiveness of stabilization orthoses for acute and chronic back pain is discussed very controversially. A study by a working group around the English physician Valle Jones JC, published in 1992, reported a reduction of the complaints. The clinical study investigated the effect of a stabilization orthosis in 216 patients with non-specific low back pain. One group was treated with orthosis, the other one received standard therapy and general tips on how to behave. The result: after just three days the patients with stabilization orthosis were already better, after three weeks 85 percent of them were able to work again without restriction. In the control group, however, this was only 67 percent. The use of painkillers was significantly lower [21] in the stabilization orthosis group over the entire trial period. A Cochrane based analysis of the available studies on stabilization orthoses for the treatment of back pain and primary/secondary prevention was published by Jellema et al. [12]. Five randomized and two non-randomized prevention studies and six therapy studies showed no effect in the preventive use of similar stabilization orthoses (which product type was investigated exactly??????). Therapeutically, there was limited evidence for each stabilization orthosis. As a recommendation, high-quality studies on the effectiveness of orthotics were called for and special emphasis was placed on adequate patient compliance [11]. Other studies investigating the benefits of comparable lumbar spine prostheses in the working process found little or no preventive benefit, with assessment criteria mostly based on questionnaires and clinical investigation. Objectively measurable parameters such as muscular strength or static parameters were not or only incompletely measured [12].

There is a better data situation with regard to EMG changes in back pain. For example, Cassisi et al. found a weakened surface EMG in the back extension area. Contrary to the common assumption, the muscles did not exhibit spasm, but rather a neuronal dysfunction [5]. This is in line with the functional results of other workgroups that found a muscular reduction in back pain [1-6]. A painful muscle cannot react adequately and develop its physiological power. According to studies by Cholewicki et al. [9], after temporary wearing of stabilization orthoses ( 3 hours a day) the stiffness of the spine increases initially. In addition, agonists in the back extension area initially switched off agonists. After a longer observation interval of 3 weeks, however, no adverse neuromuscular dysfunctions were observed [6]. The results obtained in the application monitoring allow at least some key statements. They are not in a position to answer all the questions, which would require even greater numbers of patients to be examined. In the stabilization arthroplasty groups, the complaints were statistically significantly lower than in the groups without an orthosis. The aspect that has occurred that the decrease in pain intensity in both groups is similar until the end of the first week could be seen as an effect of drug pain therapy. The positive influence of the stabilization orthosis on the musculature can only be clearly seen in the following. Without active orthotic support, tension and pain seem to stagnate. The significantly greater reduction in the amount of painkiller in the bandage group supports this assertion (Figure 9). The muscular strength in the measured movement planes improved significantly more in the stabilization orthosis groups, and it was irrelevant whether there was an additional exercise session. The general statement "regular wearing of stabilization orthoses weakens the musculature" should be refuted with this statement. The effect was particularly impressive in the extension area, as the best results were achieved in groups 3 and 4. An explanation for the improvement of muscle strength would be the better proprioceptor function in the muscle after pain relief with a resulting better response. Under pain conditions (myogelosis, facet pain, etc.) the muscular control loop is constantly disturbed and chronically strained muscles, especially in the back extension area, cannot relax. Via the abdominal press and the associated relief of the spine, the pathological control loop can be indirectly interrupted and the triggering pain is reduced. In practice, group 2 most closely corresponds to the usual treatment strategy, whereby no stabilization orthosis is often prescribed but physiotherapy and exercise are recommended. A prerequisite for optimal participation of the patient in physiotherapy is a fast and lasting freedom from pain, which can be achieved primarily by the timely use of potent pain medication. Otherwise, a patient with persistent pain will only tolerate physiotherapy or massage to a limited extent. The patients in group 1 showed the worst strength values in muscle testing, with some of them even worse than the initial examination after 6 weeks of the re-test (Figure 24).

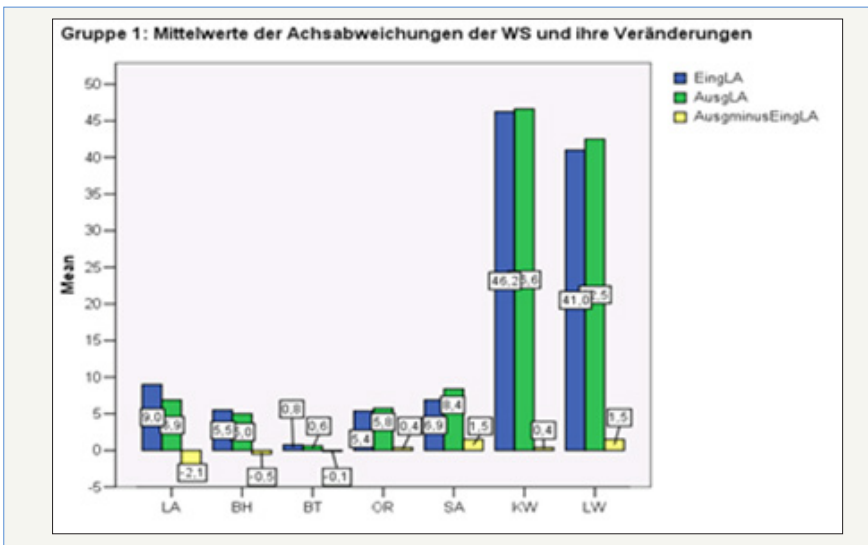

Figure 24: Overview of the measured values collected in group 1.

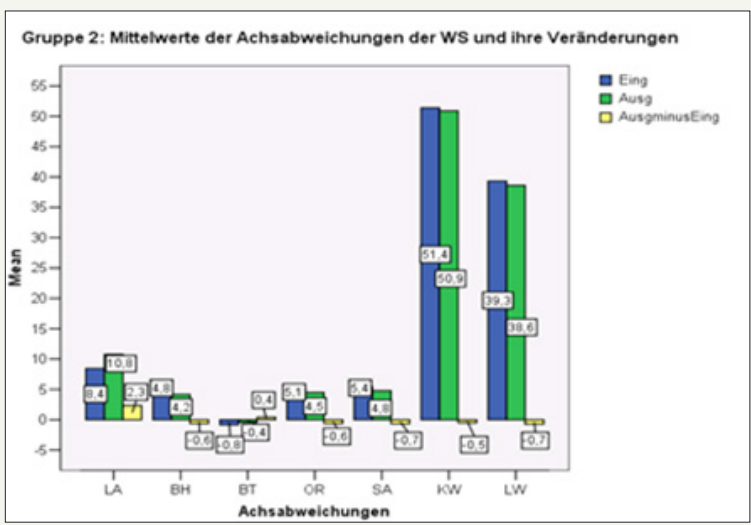

Figure 25: Overview of the measured values collected in group 2.

The vertebral column abnormal statics remain evident despite bandage and even after additional 12 times of physiotherapy. Initial functional disorders are turned into structural disorders by the body's structural disorders, which are often acquired over decades. 
Due to the decreasing elasticity of arthromucular structures, these are not so easily reversible in old age. For this reason, it is not surprising, that the prescribed 12 times physiotherapy for improving statics did not have a significant long-term effect on posture (Figure 25 ). If the incorrect posture is to be corrected effectively and in the long term, both a higher training frequency (preferably daily in combination with self-exercises of about 15-20 minutes) and special back programs are necessary. In practice, however, there are unfortunately problems with regard to patient compliance and medical prescription options. It would be desirable to rethink the general public and health insurance companies (there are currently no preventive measures available for the orthopaedic spine as part of the health insurance system), leading to a greater awareness of health issues and greater personal commitment. Ultimately, only everyone can help themselves. The physician should point out the potential possibilities and plan a therapy concept together with the patient according to the physical possibilities (Figure 26-29).

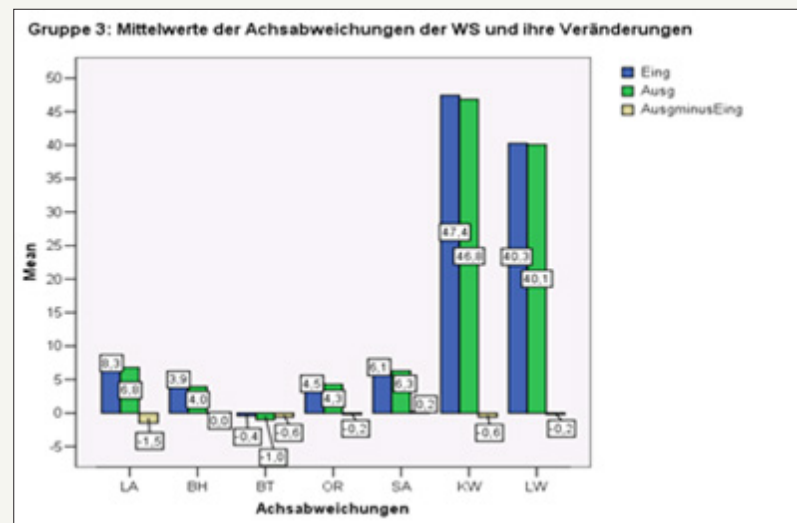

Figure 26: Overview of measured values collected in group 3 .

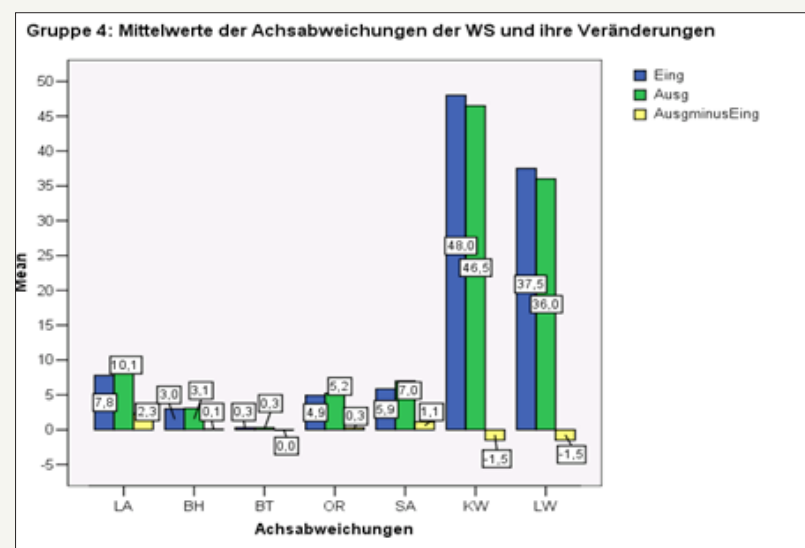

Figure 27: Overview of the measured values in group 4 In group 4 the strongest overall changes in the sagital profile were found. At both LWS and BWS baseline values improved by -1.5 . Lot deviation increased in the group. Overview of sagittal profile.

\section{Conclusion}

In summary, the ascertained muscle strength parameters and the statics analysis provide objective evidence that wearing the bandage leads to a significant increase in muscle strength, even if there is no pain. The lordosis angle obtained in the 3-D examination tends to improve after 6 weeks with the stabilization orthosis, but only in combination with physiotherapy (pos short-term effect, long-term effect only with muscle training). Uncertainties and fears among therapists and patients with regard to a worsening of muscle function due to the regular wearing of bandages should therefore be eliminated proportionately. As a general rule, bandages should be worn regularly in case of false statics and positive pain anamnesis. Especially in older age, it is usually sufficient to have one's own body weight when standing on the spot for a longer period of time, to tilt the upper body forward when brushing teeth or to lift loads unphysiologically to trigger pain. Chronic malpositions are only partially corrected when wearing the bandage, but after taking off the bandage it appears again. Only through an active life and targeted training can everyone (even or especially in old age) ensure that the bandage only has to be a temporary support with all its "ailments".

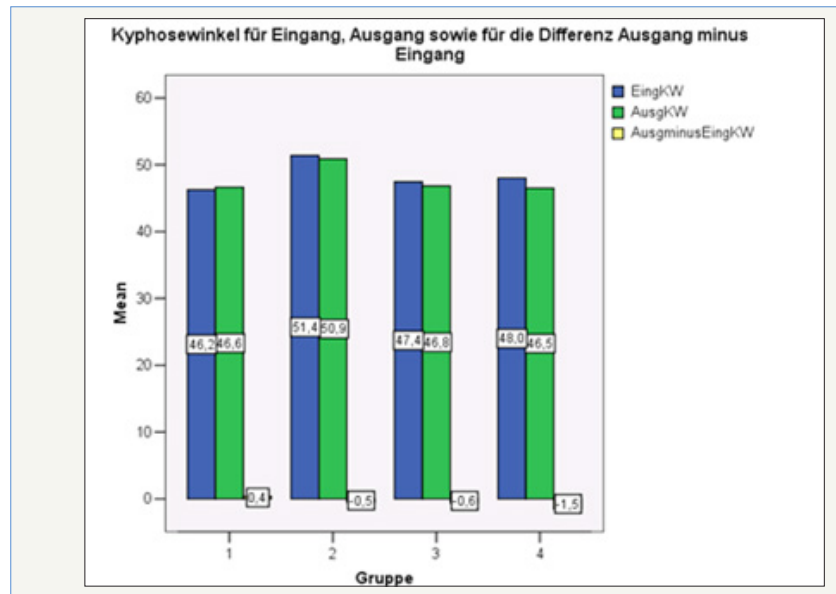

Figure 28: Change of kyphosis angle in groups 1-4 Lumbar angle: Statistically significant improvements were noted in Group 4, while a significant improvement still to be evaluated is in Group 2. A statistically non-significant deterioration occurred in group 1; the changes in group 3 are also statistically not significant.

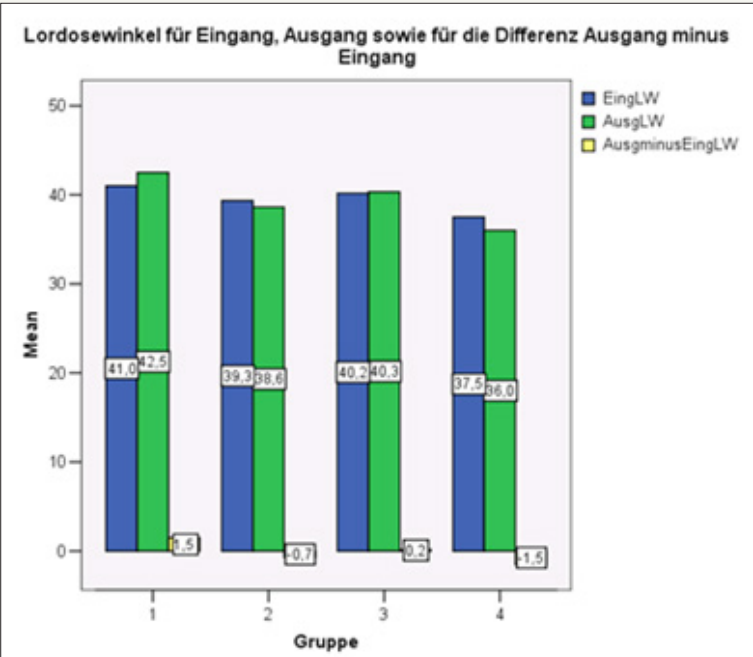

Figure 29: Changes in lordosis angle in groups 1-4 The assessment of the other individual parameters from a causality point of view proved to be very problematic, as they had a wide range of variations. 


\section{References}

1. Burdorf A (1995) Reducing random measurement error in assessing postural load on the back in epidemiologic surveys. Scand J Work Eilviron Health 21(1): 15-23.

2. Burdorf A, Naaktgeboren B, de Groot H (1993) Occupational risk factors for low back pain among sedentary workers. J Occup Med 35(12): 1213 1220 .

3. Burdorf A, Sorock G (1997) Positive and negative evidence of risk factors for back disorders. Scand J Work Environ Health 23(4): 243-256.

4. Denner A (1995) Muskuläre profile der Wirbelsäule. Bd 1 und 2, SPORT und BUCH Strauß, Köln, Germany.

5. Denner A (1998) Analyse und training der wirbelsäulenstabilisierenden Muskulatur. Springer Verlag, New York, USA, p. 1.

6. Takemasa R, Yamamoto H, Tani T (1995) Trunk muscle strength in and effect of trunk muscle exercises for patient with chronic low back pain. Spine 20(23): 2522-2530.

7. Barmer GEK Gesundheits report.

8. Jeffrey CE, Michael RE, Conner O, Michael M (1993) Trunk strength and lumbar paraspinal muscle activity during isometric exercise in chronic low-back pain patients and controls. Spine 18(2): 245-251.

9. Cholewicki J, McGill KC, Shah KR, Lee AS (2010) The effects of a threeweek use of lumbosacral orthoses on trunk muscle activity and on the muscular response to trunk perturbations. BMC Musculoskelet Disord 11: 154 .

10. Chou R, Qaseem A, Snow V, Casey D, Cross JT, et al. (2007) Diagnosis and treatment of low back pain: a joint clinical practice guideline from the american college of physicians and the American pain society. Ann Intern Med 147(7): 478-491.

11. Hayden JA, van Tulder MW, Tomlinson G (2005) Systematic review: strategies for using exercise therapy to improve outcomes in chronic low back pain. Ann Intern Med 142(9): 776-785

12. Jellema $\mathrm{P}$, van Tulder MW, van Poppel MN, Nachemson AL, Bouter LM (2001) Lumbar supports for prevention and treatment of low back pain: a systematic review within the framework of the cochrane back review group. Spine 26(4): 377-386.
13. Jellema P, Zeinstra SMB, van Poppel MN, Bernsen MDR, Koes BW (2002) Occup Med 52(6): 317-323.

14. Martimo KP, Verbeek J, Karppinen J, Furlan AD, Takala EP, et al. (2008) Effect of training and lifting equipment for preventing back pain in lifting and handling: systematic review. BMJ 336(7641): 429-431.

15. Koes BW, Hoogen HMM (1994) Efficacy of bedrest and orthoses of low backpain: a review of randomized clinical trials. Eur J Phys Med Rehabil 4(3): 86-93.

16. Kohlmann T, Deck R, Klockgether R, Raspe $H$, Brockow $T$, et al. (1994) Rückenschmerzen in der Lübecker Bevölkerung-Syndrome, Krankheitsverhalten und Versorgung. Abschlussbericht 57(4): 238-240.

17. Lühmann D, Müller V E, Raspe H (2004) Expertise im auftrag der bertelsmann-stiftung und der akademie für manuelle medizin, Universität Münster, Germany.

18. Pope MH, Philips RB, Haugh LD, Hsieh CJ, Mac Donald L, et al. (1994) A prospective randomized three week trial of spinal manipulation, TMS, massage and corset in the treatment of subacute low backpain. Spine 19(22): 2571-2577.

19. Van Poppel MNM, Koes BW, vander Ploeg T, Smid T, Bouter LM (1998) Lumbar supports and education for the prevention of low back pain in industry: a randomised controlled trial. J Am Med Assoc 279(22):17891794

20. Van Tulder MW, Jellema P, Van Poppel MNM, Nachemson AL, Bouter LM (1991) Lumbar supports for prevention and treatment of low back pain. Cochrane Database Syst Rev 3: CD001823.

21. Valle Jones JC (1992) Controlled trail of a back support (Lumbo Train) in patients with non specific back pain. Current Medical Research and Opinion 12(9): S. 604-613.

22. Zippel (1984) Hartmut Orthopädie: Leitfaden für Studierende Aufl. Verl Volk und Gesundheit.
Creative Commons Attribution 4.0 International License

For possible submissions Click Here

\section{Submit Article}

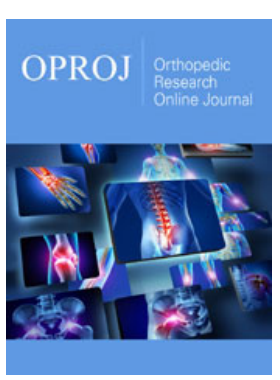

Orthopedic Research Online Journal

Benefits of Publishing with us

- High-level peer review and editorial services

- Freely accessible online immediately upon publication

- Authors retain the copyright to their work

- Licensing it under a Creative Commons license

- Visibility through different online platforms 